\title{
Libration Point Navigation Concepts Supporting the Vision for Space Exploration
}

\author{
J. Russell Carpenter; David C. Folta, Michael C. Moreau, and David A. Quinn ${ }^{\dagger}$ \\ NASA Goddard Space Flight Center, Greenbelt, MD 20771, USA.
}

\begin{abstract}
This work examines the autonomous navigation accuracy achievable for a lunar exploration trajectory from a translunar libration point lunar navigation relay satellite, augmented by signals from the Global Positioning System (GPS). We also provide a brief analysis comparing the libration point relay to lunar orbit relay architectures, and discuss some issues of GPS usage for cis-lunar trajectories.
\end{abstract}

\section{Nomenclature}

$L_{1} \quad$ Cis-lunar libration point (between Earth and Moon)

$L_{2} \quad$ Trans-lunar libration point (beyond Moon's orbit)

$L_{4}, L_{5} \quad$ Leading and trailing equilateral libration points, respectively

$\ell_{1}, \ell_{2} \quad 1575 \mathrm{MHz}$ and $1227 \mathrm{MHz}$ GPS carrier frequencies, respectively

\section{Introduction}

Farquhar ${ }^{1}$ described several libration point navigation concepts that would appear to support NASA's current exploration vision. One concept is a lunar relay satellite operating in the vicinity of Earth-Moon $L_{2}$, providing "Earth-to-lunar far-side and long-range surface-to-surface navigation and communications capability." Reference 1 lists several advantages of such a system in comparison to a lunar orbiting relay satellite constellation. Among these are one or two vs. many satellites for coverage, simplified acquisition and tracking due to very low relative motion, much longer contact times, and simpler antenna pointing. An obvious additional advantage of such a system is that uninterrupted links to Earth avoid performing critical maneuvers "in the blind." Renault and Scheeres ${ }^{2}$ have estimated that the stationkeeping cost for such a satellite, when considering the impact of navigation errors of $10 \mathrm{~km}$ and $10 \mathrm{~mm} / \mathrm{sec}$, would be on the order of $2-3 \mathrm{~m} / \mathrm{sec}$ per month, assuming maneuvers approximately every two days. Folta and Vaughn ${ }^{3}$ present a survey of stationkeeping costs for libration point orbits in the Earth-Moon system.

Another concept Farquhar described is the use of Earth-Moon $L_{1}$ for lunar orbit rendezvous, rather than low lunar orbit as was done for Apollo. Farquhar claims this technique requires only slightly higher fuel cost than low lunar orbit rendezvous for short-stay equatorial landings. More recently, Condon and Wilson ${ }^{4}$ have estimated that libration point rendezvous missions have significant advantages over lunar orbit rendezvous missions when global access, long stay times, and anytime aborts are driving requirements for human exploration missions. However, these trades, and the trade vs. lunar surface rendezvous, are significantly complicated by as yet undetermined abort requirements.

Farquhar also described an interplanetary transportation system that would use libration points as terminals for an interplanetary shuttle. This approach would offer increased operational flexibility in terms of launch windows, rendezvous, aborts, etc. in comparison to elliptical orbit transfers. More recently, other works ${ }^{5,6}$ have shown that patching together unstable trajectories departing Earth-Moon libration points with stable trajectories approaching planetary libration points may also offer lower overall fuel costs than elliptical orbit transfers. The lunar navigation infrastructure should evolve to support such concepts.

*Aerospace Engineer, Flight Dynamics Analysis Branch, Code 595, AIAA Senior Member.

$\dagger$ Aerospace Engineer, Flight Dynamics Analysis Branch, Code 595, AIAA Member. 
Another concept Farqhuar described was a deep space relay at Earth-Moon $L_{4}$ and/or $L_{5}$ that would serve as a high data rate optical navigation and communications relay satellite. The advantages in comparison to a geosynchronous relay are minimal Earth occultation, distance from large noise sources on Earth, easier pointing due to smaller relative velocity, and a large baseline for interferometry if both $L_{4}$ and $L_{5}$ are used. Such a relay could initially support lunar missions as well.

Barton et al. ${ }^{7}$ studied the use of the Global Positioning System (GPS) for navigation enroute between the earth and the moon. Assuming modest modifications that would improve GPS receiver sensitivity by approximately $10 \mathrm{~dB}$ and a high-gain directional receiver antenna, they showed that GPS signals viewed over the earth's limb would support post-translunar injection (TLI) navigation out to about half the lunar distance. They also showed GPS navigation could support a mid-course trim burn for at least several hours after TLI, but if the trim burn was more than 8 hours after TLI, there was not enough GPS information to estimate the post-burn state. This level of GPS coverage might support the $L_{1}$ lunar rendezvous scenario, especially if augmented by additional signals from NASA's Tracking and Data Relay Satellite System (TDRSS), or from navigation assets in the vicinity of the moon. More significant improvements in GPS receiver sensitivity could likely be achieved by telemetering the GPS navigation message via a distinct communications link to lunar navigation receivers, avoiding the need for the users to decode the GPS ephemerides. Such a system might support limited GPS navigation all the way to the Earth-side lunar surface.

In this work, we make a preliminary assessment of a lunar navigation infrastructure based on the concepts described by Farquhar and Barton et al. First we compare and contrast these concepts to various alternative navigation architectures for cis-lunar operations. Next, we describe calculations that establish the feasibility of using GPS for cis-lunar transfers, and possibly in support of $L_{1}$ lunar rendezvous scenarios. Finally, we illustrate some representative autonomous navigation accuracy results for a cis-lunar transfer scenario that uses GPS pseudoranges in combination with one-way Doppler measurements from an Earth-Moon $L_{2}$ Orbiter (EML2O).

\section{Architecture Analysis}

In this section we describe and compare lunar-orbiting constellations and EML2 orbiting constellations. We did not perform an exhaustive analysis to determine optimal constellation coverage. Instead, we selected what appear to be representative circular and elliptical single-coverage constellations from the literature as a starting point for our lunar orbiting constellations, and a representative class of large amplitude orbits about $L_{2}$. We also did not try to identify "frozen" lunar orbits, since these rely on properties of the lunar gravity field, and significant portions of the far-side field are currently poorly quantified due to the lack of lunar far-side tracking.

\section{Methodology}

In developing these concepts, we limited the trade space with the following guidelines: no outages during critical maneuvers and operations; navigation solutions must be computable onboard all user vehicles; navigation assets should be disposable at the end of mission; surface user latitude should be at least 75 degrees; minimum surface user elevation angle must be at least 10 degrees; a relatively small number of users must be supported in the lunar vicinity at any given time; and finally, instantaneous positioning is not required. We also assumed that an earth return vehicle remaining at $L_{1}$ during a surface stay can serve as an Earth-side communications relay if needed to avoid long light-time delays that would result from using near-Earth relays for low-latitude Earth-side operations. Clearly, an additional relay satellite orbiting $L_{1}$ could also serve this function if needed. We further assumed that all concepts would make maximal use of the Earth-orbiting GPS and TDRSS constellations. We did not explicitly study ground-based tracking from Earth since it may not support high-latitude surface operations and cannot support trans-lunar (far-side) operations, although it could clearly support cis-lunar space operations and near-side surface operations.

Figure 1 depicts our reference mission, which consists of departure from a low Earth parking orbit at 28.5 degree inclination, cis-lunar coast with lunar swingby to $L_{1}$ with at least one TLI trim burn, parking and stage separation at $L_{1}$, and descent to a high-latitude landing site. This reference mission has been selected not only as representative of a possibly typical exploration scenario, but also because it contains what we believe to be the events and mission phases that will most stringently drive navigation requirements: the TLI burn and post-TLI trim burns, $L_{1}$ station-keeping, separation and rendezvous in the $L_{1}$ vicinity, 


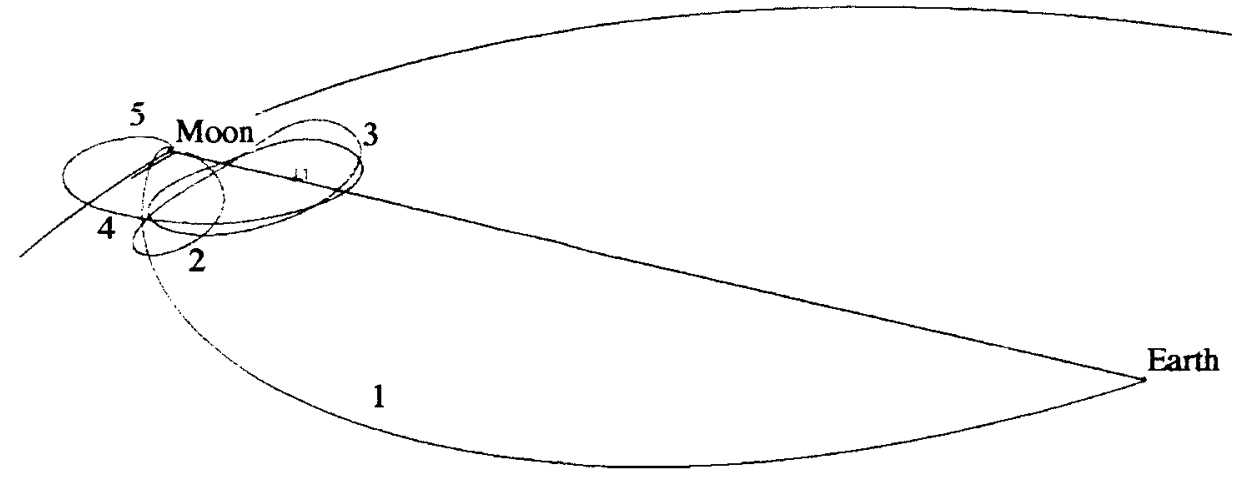

Figure 1. Representative cis-lunar trajectory viewed from above the moon's orbit around the earth, in coordinates rotating with the moon's orbit; this work primarily examines the segment labeled "1."

descent and landing to a high-latitude site, and surface operations at a high-latitude site. In this work, we focus primarily on the segment labeled " 1 " in the figure, which is the initial transit from Earth to Moon.

\section{Lunar Orbit Constellations}

As a starting point, we examined the possibility of establishing a four-satellite constellation that places three of the four spacecraft in high altitude, eccentric orbits. Constellations of this type, discussed by Draim ${ }^{8}$ for use around the earth, provide single-fold global coverage with a minimum number of satellites. The constellation Reference 8 proposes calls for three spacecraft with eccentricities of 0.13 and inclinations of 33 degrees uniformly distributed in right ascension so as to yield maximum coverage of the globe. With the periapses for such orbits in the southern hemisphere, this is an ideal means of providing continuous coverage of the land masses of the earth, which are concentrated in the northern hemisphere. For complete global coverage a fourth spacecraft is set into an equatorial orbit with a period half that of the other three. The semi-major axes of the three inclined spacecraft are determined geometrically by the need to maximize the coverage. If we scale this by the radius of the central body, we find that the $106,440 \mathrm{~km}$ semi-major axis called for at the earth translates into a semi-major axis of $29,000 \mathrm{~km}$ at the Moon. The problem here is that the coverage geometry scales with distance while the gravitational attraction of the central body scales with mass, which is a function of volume. Where the earth with its relatively large mass is the central body, this is not a problem since third body perturbations from the moon and sun have little effect on the long term stability of the constellation. When this geometry is scaled down for the moon however, we find that the low gravity of the Moon combined with the relatively large third body perturbations from the earth prevent the orbits needed for a constellation of this type about the moon from being stable. Further we find that if we were to reduce the semi-major axes of the constellation to address this stability issue, gaps in the coverage open up. This eliminated this particular elliptical orbit constellation from further consideration for global coverage at the moon.

Next, we looked at how six satellites in a constellation of a type discussed for Earth coverage by Lang and $\mathrm{Meyer}^{9}$ could be applied to the problem of continuous global lunar coverage. Reference 9 claims that this constellation provides continuous global coverage by at least one satellite at lower altitudes for a given minimum elevation constraint than any other six satellite constellation. This constellation, which Figure 2 illustrates, employs four spacecraft in circular orbits, all at 58.9 degree inclinations, distributed in right ascension at 90 degre intervals. Two spacecraft in opposing circular equatorial orbits fill the coverage gaps left by the first four. The result is a constellation with a global coverage pattern that repeats at a semimajor-axis-dependent frequency. When the minimum elevation for a user anywhere on the lunar surface is 10 degrees, a Moon-centered constellation with a semi-major axis of $6214 \mathrm{~km}$ and a period of 12.2 hours results. Such low, circular orbits about the moon turn out to be stable in the long term but can be costly to establish. For computing the establishment cost, we assumed the six spacecraft were sent to the moon with two launches, each carrying three spacecraft. In this scenario, each triad would establish a lunar orbit before separation from the carrier spacecraft.

Since the trade space of possible Moon-centered elliptical constellations is so expansive, we developed a 


\section{Circular (Lang-Meyer) Configuration}

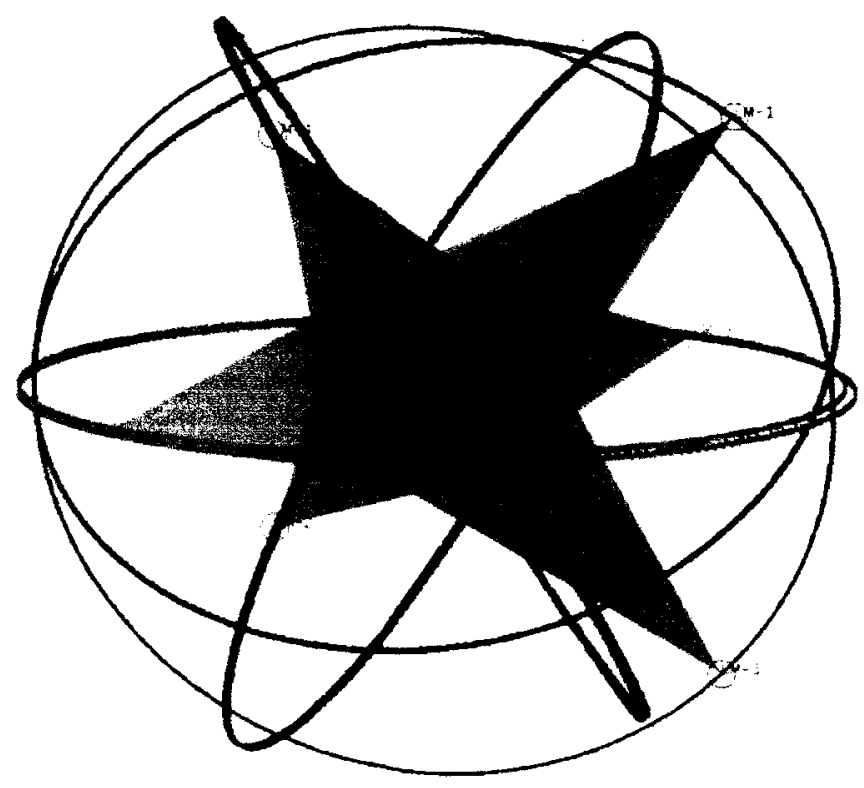

Figure 2. Circular lunar orbit constellation, based on the work of Lang and Meyer.

\section{Elliptical (Modified Lang-Meyer) Configuration}

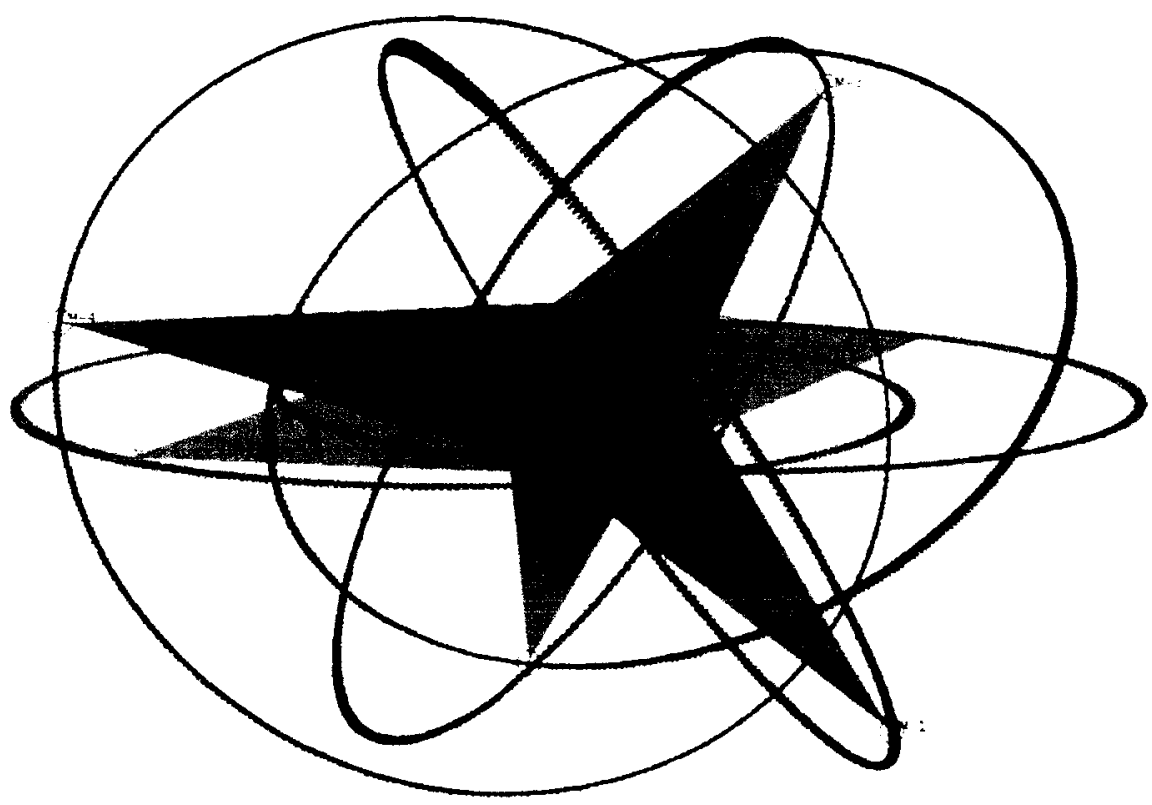

Figure 3. Elliptical lunar orbit constellation, based on extension of the Lang and Meyer orbits. 
representative case. In this case, which Figure 3 shows, we employed the orientations of the Lang and Meyer constellation using eccentric rather than circular orbits. We set periapsis to the minimum radius that results in a greater than 10 degree minimum elevation for a user on the lunar surface. We set an outer boundary for apoapsis at 10,000 km; we observed that higher or bits tended to be less stable, but we did not perform an exhaustive analysis. The semi-major axis of the subsequent orbits turned out to be $8125 \mathrm{~km}$ with a period of 18.2 hours and an eccentricity of 0.23 . Establishing the eccentric orbits may be accomplished by approaching the lunar surface at $100 \mathrm{~km}$ altitude and executing a lunar orbit insertion (LOI) burn that sets the apoapsis of the desired orbit at $9993.75 \mathrm{~km}$. Once the spacecraft moves to apoapsis, a burn would be executed raising periapsis to its final value of $6256.25 \mathrm{~km}$. We found that the cost of establishing such stable eccentric orbits in the Lang and Meyer orientations were marginally more expensive than the unstable high altitude circular orbits, but considerably less expensive than the highly stable low altitude circular orbits. Inclination changes done at apoapsis are much less expensive for the eccentric constellation than for the stable low altitude constellation. In this sense, this eccentric version of the Lang and Meyer constellation may be used as representative of the expense of establishing a useful, Moon-centered global coverage capability. Here again, we computed the establishment cost assuming that two TLI vehicles are used to get two triads of spacecraft to the moon, which upon LOI separate to form the constellation.

\section{Earth-Moon $L_{2}$ Orbiter Constellation}

Next, we sought to define a trajectory for one or more Earth-Moon $L_{2}$ Orbiters that would provide coverage for high-latitude sites on the moon. Figure 4 depicts one possible configuration, which is a compromise between minimizing station-keeping costs and maximizing the out-of-plane amplitude of the trajectory around $L_{2}$ so as to provide highlatitude coverage on the moon. This configuration consists of a pair of large Lissajous orbits that have opposite inclinations in the plane normal to the moon's velocity. Although a single satellite in one of the orbits will provide continuous coverage for nearly the entire lunar far-side hemisphere, in order to get continuous coverage above 10 degree elevar

Table 1. Phases of the EML2 orbiters, which are described with respect to the maximum elevation above the lunar orbit plane of a virtual satellite at the initial simulation epoch of 17 April 2008, 0.0 UT. For this coverage pattern, satellites 1 and 3 are in the blue "upper" $L_{2}$ orbit, and satellites 2 and 4 are in the red "lower" orbit, which Figure 4 depicts.

tion at both lunar poles requires two satellites in

\begin{tabular}{|c|c|}
\hline Satellite & Phase[days] \\
\hline 1 & 1.94 \\
2 & -1.94 \\
3 & -5.81 \\
4 & 5.81 \\
\hline
\end{tabular}
each orbit. Figure 5 shows the elevation angles to the constellation of EML2 Orbiters that a station located directly at either pole of a perfectly spherical Moon would see, ignoring the physical libration of the lunar pole (which is only a few thousandths of a degree). Table 1 lists the phases of the satellites in the two $L_{2}$ orbits. Note that these orbits have periods of about 15.5 days. Front side lunar coverage would be provided by near-Earth space networks such as TDRSS, Earth ground networks, or possibly by an Earth return vehicle stationkeeping near $L_{1}$. For estimating the establishment cost, we assumed the four spacecraft would be sent to the moon with two launches, each carrying two spacecraft.

\section{Architecture Comparison}

Table 2 compares the architecture concepts we finally considered. Definitions of the table's notation are as follows: $n$ is the number of satellites required for global coverage, $\Delta V_{e}$ is the establishment cost (including both Earth departure and final orbit injection), $\Delta V_{d}$ is the cost to dispose of the satellite, and $L_{s}$ is the maximum space loss, computed assuming maximum range from a polar site at zero elevation, and a frequency of $1.575 \mathrm{GHz}$. In deriving estimates for the $\Delta V$, we did not consider navigation or maneuver execution errors, or finite burn losses. To estimate the disposal $\Delta V$, we chose the lower cost between impacting the moon or escaping from the lunar vicinity (we expect that zero disposal cost will be associated with the $L_{2}$ concepts since by merely ending station-keeping, they will escape the lunar vicinity). We did not draw any conclusions regarding stationkeeping costs, since this will depend very strongly on as yet undetermined requirements. Preliminary investigations indicate however that the lunar orbits we examined are stable over at least a one year timeframe. The libration orbits will obviously require stationkeeping maneuvers, likely as often as 


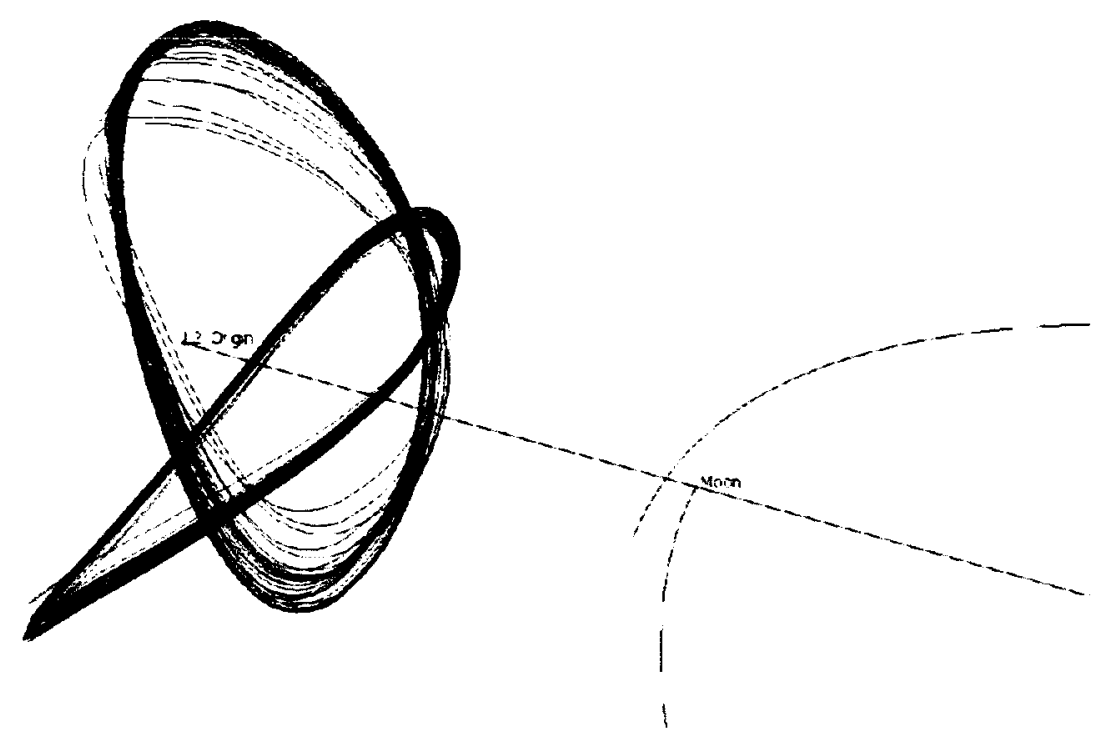

Figure 4. Earth-Moon $L_{2}$ Orbiters' trajectories. The direction of the moon's motion is toward the viewer, and the earth is to the right of the scene. The amplitudes of the orbits in the direction normal to the moon's orbit plane are approximately $25,000 \mathrm{~km}$, and they extend approximately $50,000 \mathrm{~km}$ on either side of the Earth-Moon- $L_{2}$ line. The amplitude of the motion toward and away from the moon covers about 25,000 km.
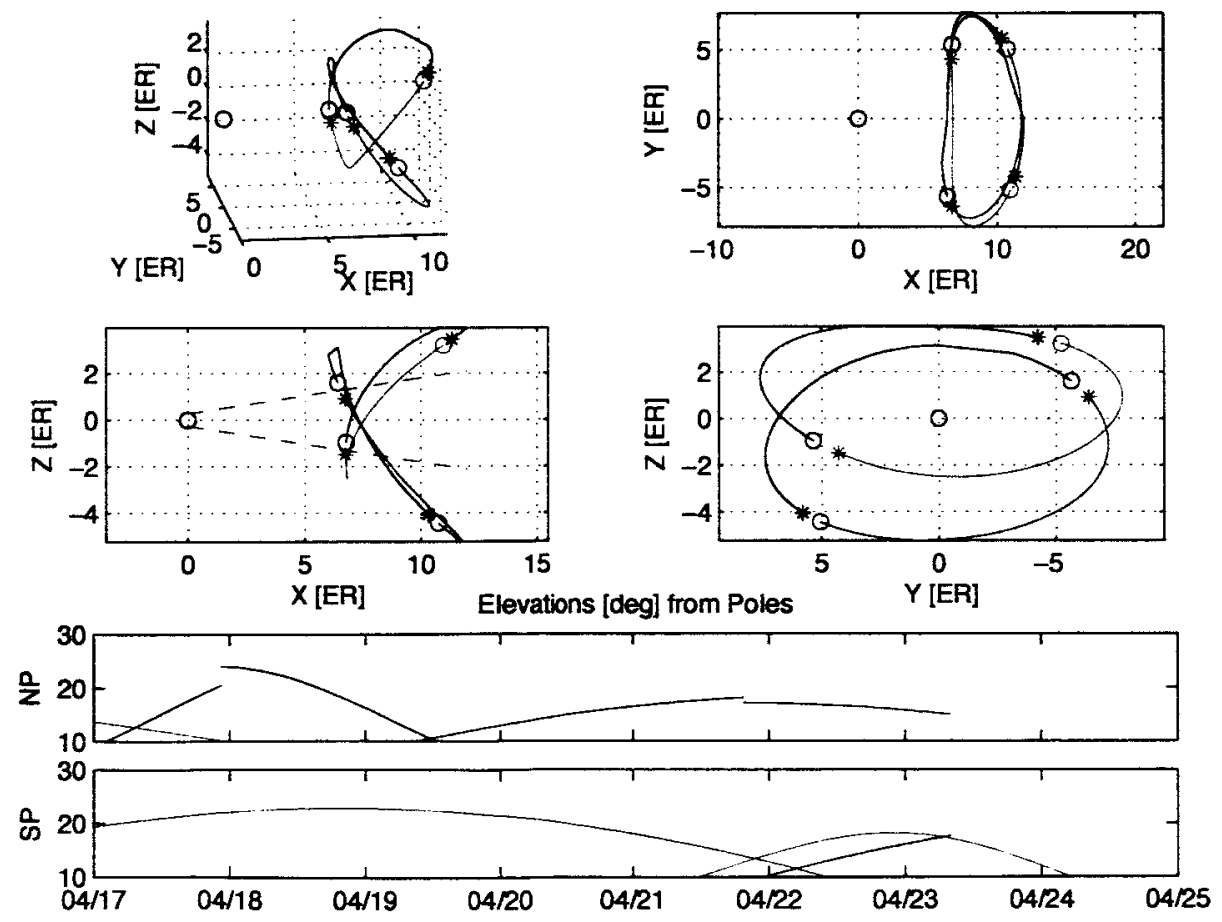

Figure 5. Plan view of $L_{2}$ Orbiters' trajectories along with elevations from stations at lunar poles, assuming alignment of Moon's axis with Moon's orbit's angular momentum. For this coverage pattern, satellites 1 and 3 are in the blue "upper" $L_{2}$ orbit, and satellites 2 and 4 are in the red "lower" orbit. The green dashed lines in the $X-Z$ plot indicate $10 \mathrm{deg}$. elevations from the lunar poles. The discontinuities in the trajectories are locations where bi-weekly stationkeeping maneuvers would need to be performed. 
every revolution (i.e. on the order of every two weeks). Based on the work of Reference 3, we estimate a stationkeeping cost for these types of orbits at around 300 meters per second per year. In any case, whatever $\Delta V$ advantage the EML2O concept may have over lunar orbiters must be offset against a maximum space loss penalty of approximately $20 \mathrm{~dB}$. However, the maximum space loss is less than $10 \mathrm{~dB}$ worse than for a geosynchronous Earth orbit (GEO) satellite, and the minimum space loss for the EML2O concept is only about $2 \mathrm{~dB}$ worse than for a GEO satellite. As a result of this brief and incomplete survey, we believe that a case can be made for lunar orbiting constellations in support of long-term human and robotic lunar exploration, but we believe that the EML2 Orbiter concept provides a great deal of capability with minimal investment as a starting point for a comprehensive lunar and planetary navigation and communications infrastructure beyond near Earth orbit.

Table 2. Comparison of representative architecture options described in the text; units of $\Delta V$ are $\mathrm{km} / \mathrm{sec}$; units of space loss are dB. Note that $\Delta V_{e}$ includes departure from low Earth orbit, and both $\Delta V$ columns represent rough estimates of the total cost for the entire constellation, exclusive of trajectory knowledge and control errors.

\begin{tabular}{|l|c|c|c|c|}
\hline Concept & $n$ & $\Delta V_{e}$ & $\Delta V_{d}$ & $L_{s}$ \\
\hline Circular lunar orbits & 6 & 13.7 & 2.2 & -172 \\
Elliptical lunar orbits & 6 & 11.4 & 2.3 & -176 \\
Large lissajous at $L_{2}$ & 4 & 8.7 & 0 & -194 \\
\hline
\end{tabular}

\section{GPS Signal Availability Analysis}

In this section, we evaluate the GPS signal availability over a four day time span covering the Earth-Moon transit segment "1" in Figure 1.

\section{Methodology}

As a baseline case, we considered a GPS receiver with an acquisition/tracking sensitivity of $25 \mathrm{~dB}-\mathrm{Hz}$ and a $10 \mathrm{~dB}$ receiving antenna. The $25 \mathrm{~dB}-\mathrm{Hz}$ threshold corresponds to a minimum ambient power of approximately $-184 \mathrm{~dB}$ Watts $(\mathrm{dBW})$, or received power at the output of the antenna of $-174 \mathrm{dBW}$. This performance is consistent with a state of the art GPS receiver implementing modern weak signal tracking techniques. ${ }^{10}$ We also examined cases that assumed acquisition and tracking thresholds of $18 \mathrm{~dB}-\mathrm{Hz}$ and $11 \mathrm{~dB}-\mathrm{Hz}$. The former case is probably at or just beyond the limit of current state of the art weak-signal GPS technology to decode the GPS navigation message (broadcast ephemeris). The latter case is probably at or just beyond the limit of current state of the art weak-signal GPS technology to acquire and track the GPS signals at all. In this case, the receiver would require an alternative source of ephemeris information for the GPS satellites, which limits its autonomy.

The modeled GPS link budget includes realistic antenna gain patterns for the GPS satellites and receiver, free space propagation losses, and antenna/receiver noise models to evaluate signal levels reaching the receiver and corresponding carrier to noise spectral density. Geometric obstruction of GPS signals with respect to the earth is evaluated based on a constant $6378.14 \mathrm{~km}$ Earth radius plus a $50 \mathrm{~km}$ atmosphere mask. A single frequency $\ell_{1}$ coarse/acquistion code GPS receiver was assumed, and GPS transmitted power levels were based on the Block II/IIA GPS satellites, transmitting at typical power levels, which are approximately $1.5 \mathrm{~dB}$ above minimum specified levels. The GPS constellation was simulated based on a nominal 27 satellites, six plane configuration.

\section{Results}

Figure 6 illustrates representative GPS signal availability for the three cases described above. Figure 7 shows the corresponding ambient power and carrier to noise ratio $\left(C / N_{0}\right)$ levels for signals. Considering the baseline $25 \mathrm{~dB}-\mathrm{Hz}$ case, there is nearly continuous GPS coverage with frequently two or more satellites in view during the first half day after the TLI burn, out to about 18 Earth radii $\left(R_{E}\right)$ from the earth. This provides important observations in support of a possible correction burn that would be performed in this 
timeframe. Peak GPS signal levels are approximately $38 \mathrm{~dB}-\mathrm{Hz}$, and the spacecraft is already at a distance from the earth above which any GPS side lobe signals (greater than 25 degrees off transmitting GPS satellite nadir) extend above $25 \mathrm{~dB}-\mathrm{Hz}$. From this point onward there will never be more than three satellites visible simultaneously, and typically no more than two.

Figures 8 and 9 provide a closer look at the GPS signals available in the vicinity of the Earth-Moon $L_{1}$ point, at a range of approximately $50 R_{E}$ and 2.4 days ( 58 hours) elapsed time. For the $25 \mathrm{~dB}-\mathrm{Hz}$ case (Figure 8) passes that are interrupted because the GPS satellite sets behind the earth are short, about 10-15 minutes on either side of the earth's limb. For GPS satellites in which the earth does not obstruct the line of sight, the passes are typically 50-60 minutes in duration. Peak signal levels do not exceed $31 \mathrm{~dB}-\mathrm{Hz}$, and there is usually only a single GPS satellite visible at one time. The longest outage (no satellites available) was approximately 90 minutes. Figure 9 shows the data for an $18 \mathrm{~dB}-\mathrm{Hz}$ sensitivity. The increased sensitivity means side lobe signals are available at higher altitudes (although not out to $L_{1}$ ), which ultimately reduces the duration of outages. In the vicinity of the moon, peak GPS signals levels are approximately $29 \mathrm{~dB}-$ $\mathrm{Hz}$, but overall GPS signal availability is very similar to availability near $L_{1}$. As a general approximation, path losses are approximately $15-16 \mathrm{~dB}$ larger than GEO in the region from the $L_{1}$ point to the lunar surface. Referring back to Figure 6, note that the $11 \mathrm{~dB}-\mathrm{Hz}$ case appears to provide nearly continuous GPS coverage all the way out to the lunar surface. These results may be overly optimistic as they are based on tracking GPS side lobe signals out to 60 degrees off the GPS satellite nadir, and the actual side lobe power may deviate significantly from the modeled pattern beyond 30-40 degrees off nadir. The improved GPS availability indicated in the 18 and $11 \mathrm{~dB}-\mathrm{Hz}$ cases could nevertheless be realized using an even more directional receiving antenna. The modeled $10 \mathrm{~dB}$ antenna has a $3 \mathrm{~dB}$ beamwidth of about 20 degrees (half angle), but in the vicinity of $L_{1}$, all of the GPS signals are received within a 5 degree cone (half angle) centered around the earth.

A number of factors were examined to assess the sensitivity of the GPS availability to some of the assumptions used above. The simulation was repeated using transmitted power levels and antenna gain patterns for the modernized Block IIR-M GPS vehicles, and for the new civil "L2C" signal on the GPS $\ell_{2}$ carrier. GPS coverage was not significantly different for either of these cases. The new $\ell_{2}$ signal may actually have some advantages for weak signal tracking however, because it has a signal component that is not modulated with data allowing longer coherent integration times. ${ }^{11}$ Additionally, the GPS coverage is dependent on the geometry of the GPS constellation with respect to the outbound trajectory of the receiver. A number of different outbound trajectories with different epochs were examined, and for the $25 \mathrm{~dB}-\mathrm{Hz}$ sensitivity, GPS satellites from only two of the six orbital planes were typically visible. In the GPS constellation, each plane may contain 4-6 satellites, so the peak number of satellites visible and maximum outages vary depending on which planes of GPS satellites are observed.

\section{Navigation Analysis}

In this section, we describe our methodology and results for obtaining representative estimates of the autonomous navigation accuracy for a user vehicle on segment " 1 " of the reference trajectory Figure 1 depicts.

\section{Methodology}

We performed the navigation analysis using the GPS Enhanced Onboard Navigation System (GEONS). GEONS is an extended Kalman filter ${ }^{12}$ with physically realistic process noise models ${ }^{13,14}$ and a factored covariance matrix. ${ }^{15}$ Its measurement model may include standard and singly-differenced GPS ${ }^{16}$ and GPS Wide Area Augmentation System (WAAS) data, intersatellite crosslink data, one-way forward-link Doppler from ground stations or TDRSS, and line-of-sight and angular measurements to celestial objects ${ }^{17}$ and other spacecraft. The GEONS state vector may consist of absolute or relative position and velocity vectors in the mean equator and equinox of J2000.0 coordinate system, ${ }^{18}$ empirical drag and solar radiation pressure coefficient corrections, and measurement biases for all data types. GEONS uses the Joint Gravity Model-2 (JGM-2), ${ }^{19}$ with coefficients up to degree and order 30 , and models solar, lunar, and planetary point mass gravity using either the Van Flandern ${ }^{20}$ or Moshier ${ }^{21}$ analytical methods. GEONS has an analytic representation of the Harris-Priester ${ }^{22}$ atmospheric density model, and a simple solar radiation pressure (SRP) model, similar to that described in Vallado, ${ }^{23}$ that assumes a cylindrical Earth shadow. The filter integrates 

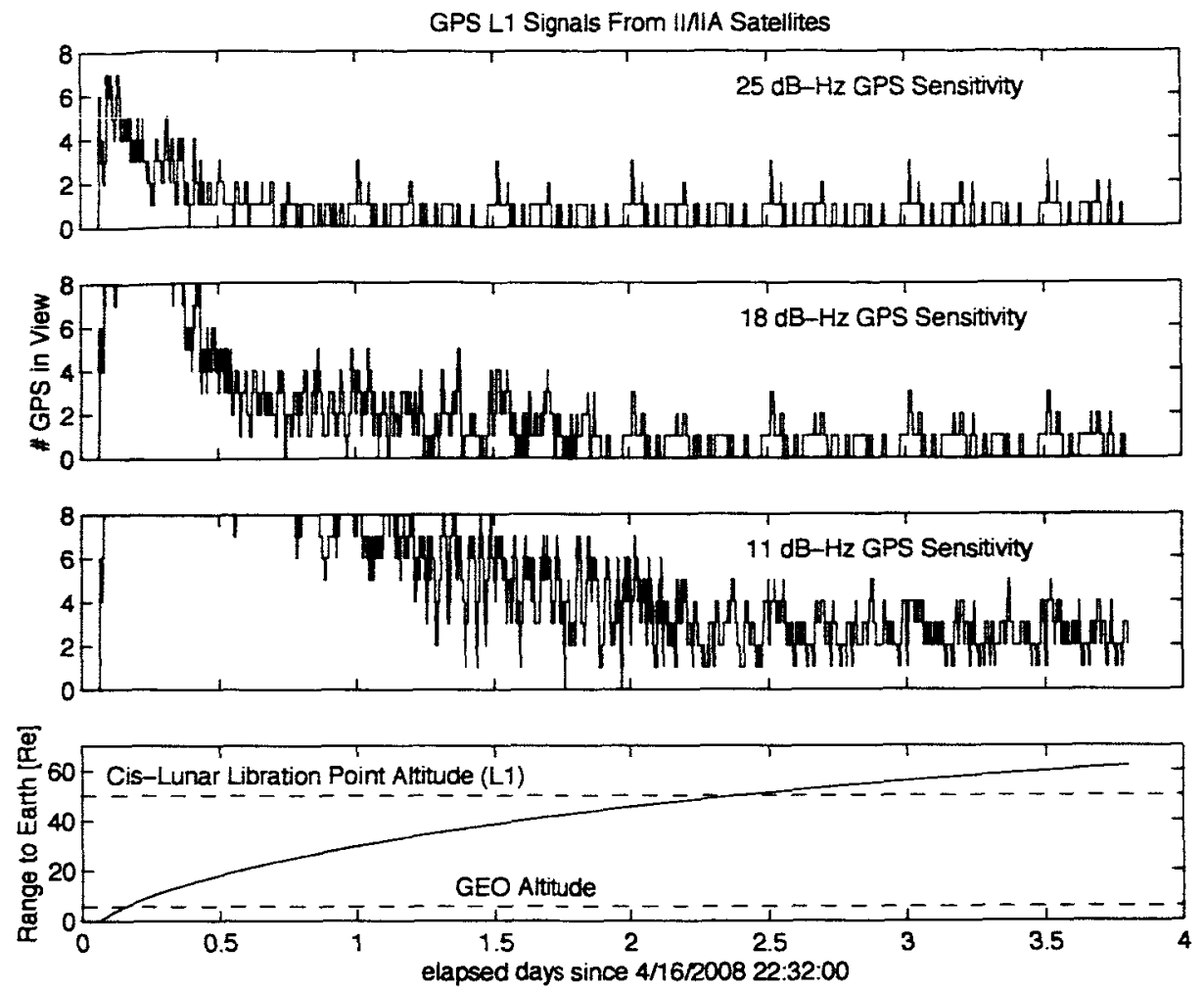

Figure 6. GPS signal availability on lunar transfer trajectory for three different receiver sensitivities.
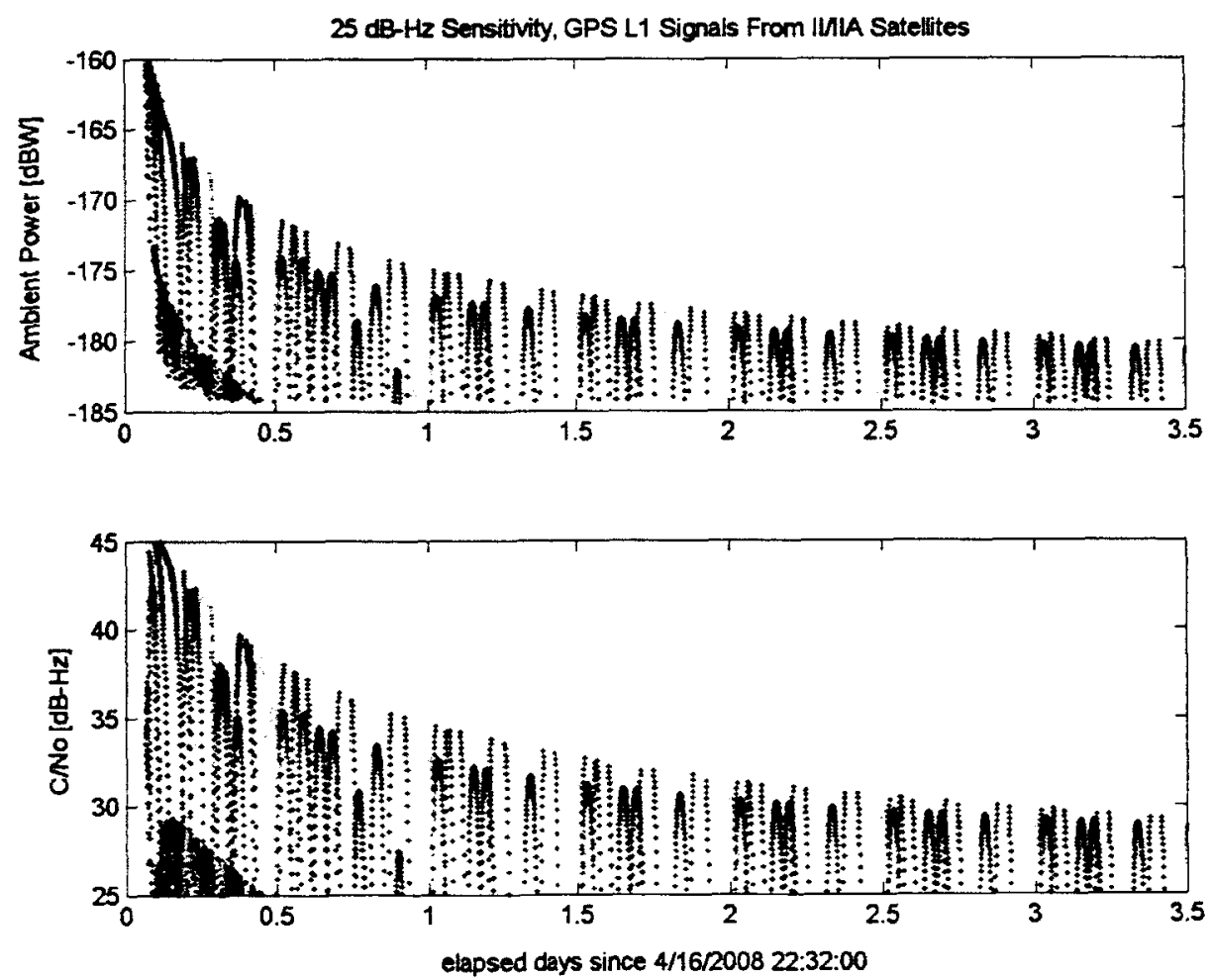

Figure 7. GPS power levels on lunar transfer trajectory. 

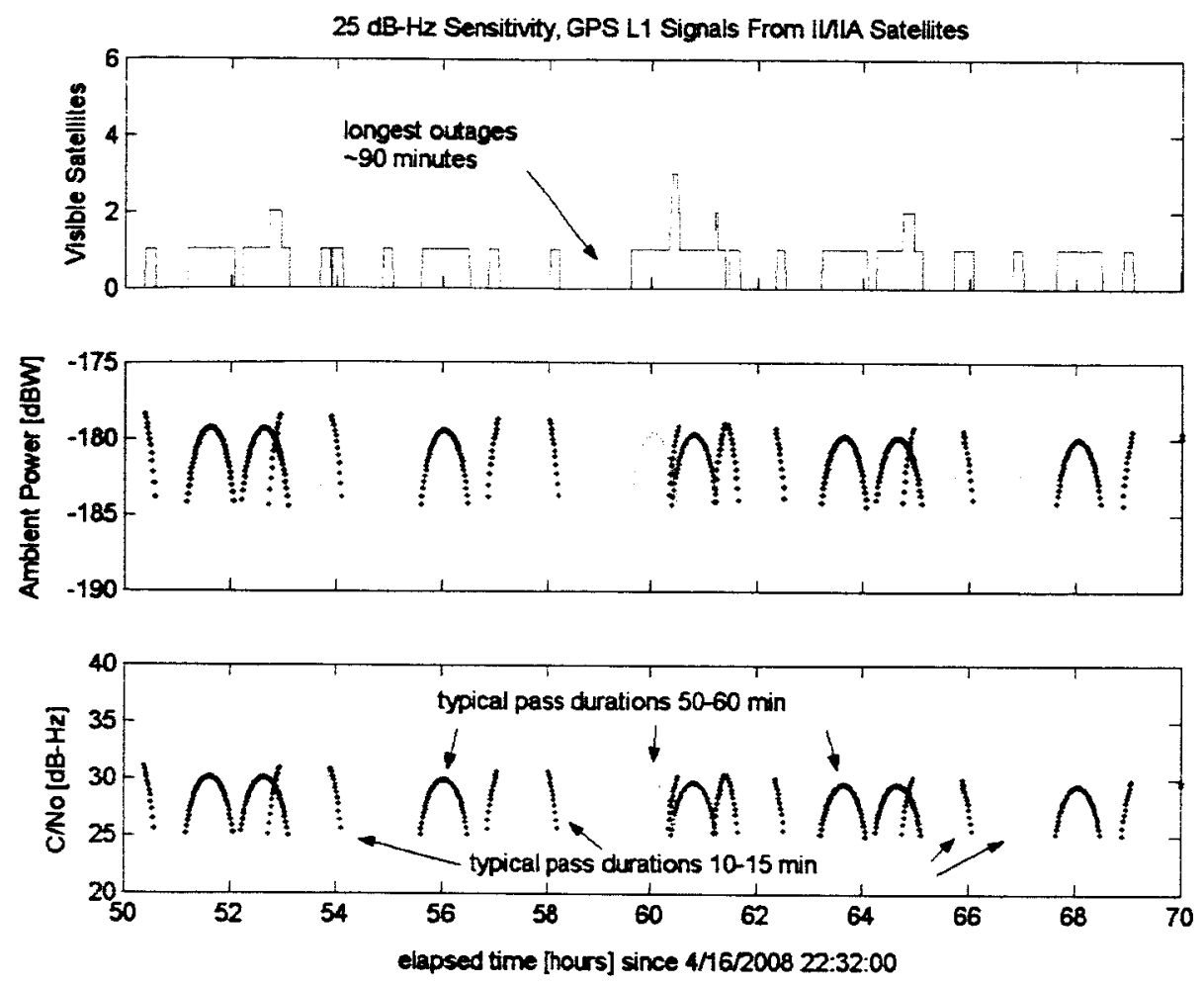

Figure 8. GPS signals above $25 \mathrm{~dB}-\mathrm{Hz}$ near the Earth-Moon $L_{1}$ point.
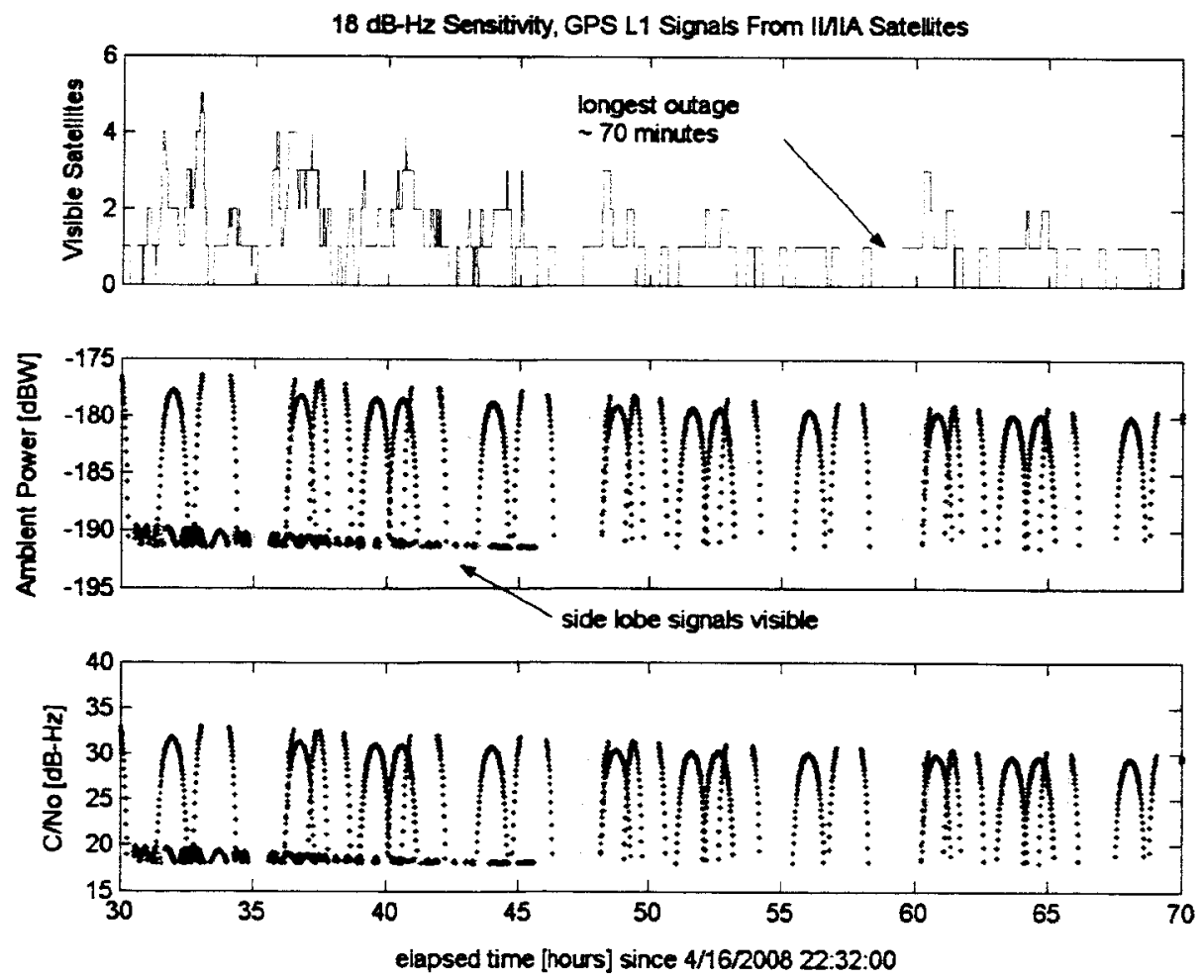

Figure 9. GPS signals above $18 \mathrm{~dB}-\mathrm{Hz}$ near the Earth-Moon $L_{1}$ point. 
the equations of motion with either 4th- or 8th-order Runge-Kutta methods, and measured accelerations may be incorporated. The state transition matrix is propagated with a semi-analytic formulation that includes Earth point mass and $J_{2}$, atmospheric drag, and solar radiation pressure partial derivatives.

For this analysis, we simulated measurements with a truth ephemeris that we generated from initiai conditions just after the cis-lunar injection departure maneuver from low Earth orbit. The truth model parameters were as follows: 30x30 JGM2 Earth gravity; point-mass Moon gravity; Moshier analytical ephemerides for the Moon, Sun, Venus, Mars, Jupiter, and Saturn; 4.51 square meters and 100 kilograms spacecraft area and mass; spacecraft drag coefficient of 2.0; spacecraft SRP coefficient of 1.1886 , with 1371 Watts per square meter assumed as the solar flux; and Allan variance parameters for the receiver clock typical of an ovenstabilized crystal oscillator $\left(h_{0}=2.4 \times 10^{-22} \mathrm{sec}\right.$, and $h_{-2}=8.0 \times 10^{-28} \mathrm{sec}^{-1}$ ). To numerically integrate the equations of motion, the truth model used an 8th-order fixed-step Runge-Kutta integrator with 3rd-body forces and rotation matrices evaluated at each function call, with a time step of $60 \mathrm{sec}$. For the simulated GPS pseudorange measurements, we added white Gaussian noise with standard deviation of 2 meters for signals stronger that $40 \mathrm{~dB}-\mathrm{Hz}$, and 10 meters for weaker signals. We simulated GPS transmitter clock and ephemeris errors using the "Lear 4 " 24 model with 2 meter standard deviation, and used a curve fit of an averaged electron density profile for ionosphere errors. To the $1575 \mathrm{MHz}$ Doppler data from the EML2 Orbiter, we added white Gaussian noise with standard deviation of $20 \mathrm{mHz}$.

We used a somewhat simpler set of model parameters in the navigation filter: $8 \times 8$ JGM2 Earth gravity; zero drag; and Allan variance parameters for the receiver clock typical of an temperature-stabilized crystal oscillator $\left(h_{0}=3 \times 10^{-14} \mathrm{sec}\right.$, and $\left.h_{-2}=8.4 \times 10^{-20} \mathrm{sec}^{-1}\right)$; all other parameters were the same as the truth model, except that we added zero-mean Gaussian random position, velocity, clock bias, and clock drift initial condition errors and a random walk SRP bias to the filter. The standard deviations of the initial errors were, for position: 31.6 meters in the radial and crosstrack directions, and 100 meters in-track; for velocity: $10 \mathrm{~cm} / \mathrm{sec}$ in all directions; for clock bias and drift: 100 meters and $10 \mathrm{~cm} / \mathrm{sec}$; and for SRP bias: $0.01 \%$. We assumed correlation coefficients of -0.95 between initial radial position and along-track velocity, and between initial along-track position and radial velocity. We computed a Gaussian random initial state perturbation for the filter using a Cholesky decomposition of the initial covariance. To account for dynamic model simplification, we also added random walk acceleration process noise, with root power spectral densities $\left(\mathrm{m} / \mathrm{sec}^{3 / 2}\right)$ of $1 \times 10^{-5}$ radial and $1 \times 10^{-4}$ cross-track. For the SRP bias, the root noise density was $1 \times 10^{-5} \mathrm{sec}^{-1 / 2}$. The filter assumed a 10 meter standard deviation for the GPS pseudorange measurement noise, and a $600 \mathrm{mHz}$ standard deviation for the EML2 Orbiter Doppler noise. The filter processed all available measurements once per minute, but it ignored GPS signals that passed less than $1000 \mathrm{~km}$ above the earth's limb. Filter tuning and monte carlo analysis of the filter performance were beyond the scope of our study, so one should view our results as representative of the kinds of accuracies that might be achieved, rather than as a definitive evaluation of the achievable performance.

\section{Results}

Figures $10-13$ plot the results of the filtering investigation. In Figure 10, the upper subplot shows the GPS pseudorange residuals, surrounded by the three-sigma formal error envelope of the residuals derived from mapping the filter's covariance. The middle subplot shows the one-way Doppler residuals from the EML2 orbiter and its formal error envelope. Both of these subplots also show the filter's residual ratios along the bottom edge, with a scale on the right. These figures indicate that the filter's residuals are generally zero-mean and uncorrelated, that GPS pseudorange residuals are generally below a few tens of meters, and that EML2O Doppler residuals are generally below a few tens of milliHertz, with occasionally large Doppler residuals that exceed the filter's six-sigma edit ratio test. We believe these large residuals are due to truncation differences between our quadruple-precision data simulator and the double-precision wordlength used in GEONS. The bottom subplot indicates the total number of contacts to both GPS and the EML2 orbiter. Since the EML2 orbiter is always visible, this plot shows that after the first half-day, only zero to three three GPS satellites are visible. Note that the GPS ephemeris we used in the navigation analysis was based on an actual current broadcast ephemeris set, rather than the nominal ephemeris set used for the visibility analysis above. As a result, one may note differences in the visibility at any given time, although the overall visibility statistics do not significantly differ.

Figures 11 and 12 show collections of position- and velocity-related error statistics. In both these figures, the first three subplots show the errors projected onto local coordinates that are aligned with the radial and cross-track directions. The "out-of-plane crosstrack" direction is co-linear with the two-body angular 

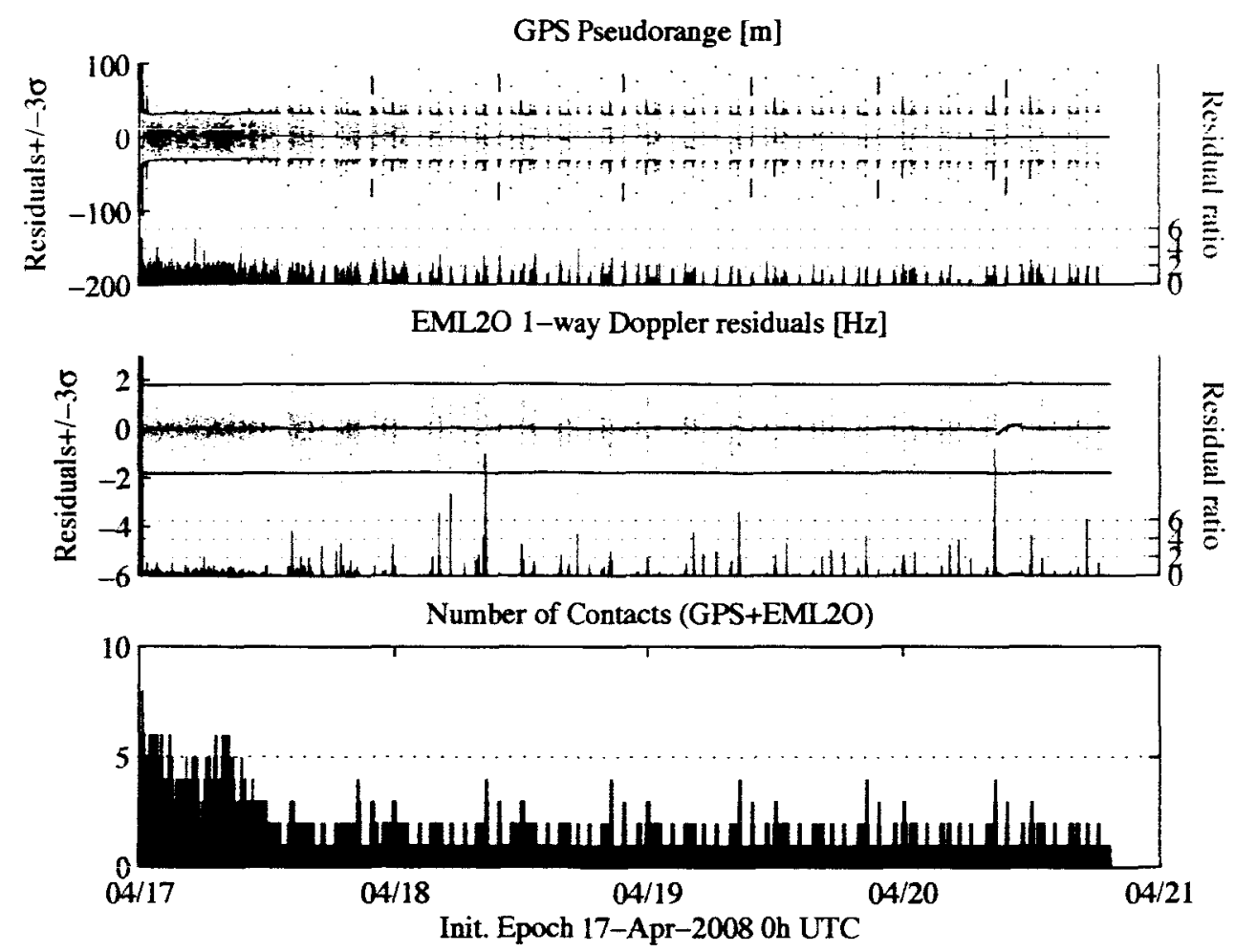

Figure 10. Measurement information processed by the filter.

momentum of the receiver, and the "in-plane crosstrack" direction completes a right-handed system with the other two directions. The fourth subplots show the position and velocity magnitude errors, and Figure 11 also plots the semi-major axis error. Both these figures also plot a formal error envelope derived from the filter's covariance matrix. Since these are single-case results, the value of the actual errors in predicting real-world performance is somewhat reduced. Instead, one may view the formal error envelope as having the value of a linear covariance analysis, that is, as guideline of the range of accuracy that might be achievable. In this sense, the accuracies of better than $1 \mathrm{~km}$ and $5 \mathrm{~cm} / \mathrm{sec}$ are quite promising in comparison with the trans-lunar post-maneuver results on the order of kilometers and dozens of centimeters per second that Beckman and Conch ${ }^{25}$ achieved during Lunar Prospector using two-way Doppler from Deep Space Network 34- and 26-meter tracking sites. It should be noted that the real-world results of Reference 25 were achieved in the face of all the constraints posed by an operational environment, including most notably imperfectly performed maneuvers that this study did not address.

Figure 13 shows a collection of the bias error estimates of the filter. The first two subplots show clock bias and drift, and the lower subplot shows the correction to the SRP coefficient. These plots show that, despite the assumption of an OCXO, the clock errors are comparable in size to the position and velocity errors. Although it is difficult to discern from the lower subplot, we did verify that the SRP correction is observable; however we did not find the filter to be very sensitive to even much larger unknown SRP biases. For this reason, we chose a filter tuning that allows the filter to consider the uncertainty in this parameter in its gain computation, but does not actually result in any significant updates to the parameter.

We also examined cases with improved GPS receiver sensitivity, using both $18 \mathrm{~dB}-\mathrm{Hz}$ and $11 \mathrm{~dB}-\mathrm{Hz}$ acquisition and tracking thresholds. Although these cases significantly arrested the decline in GPS coverage as distance increased from the earth, we found that none of the GPS-only scenerios had consistently stable results beyond $L_{1}$. The primary difficulty with these cases is that the clock states become highly correlated with the radial error states, as a result of a combination of poor geometry and poor visibility. 
Position Errors $[\mathrm{km}]$ (Actual $+/-2 \sigma$ formal)
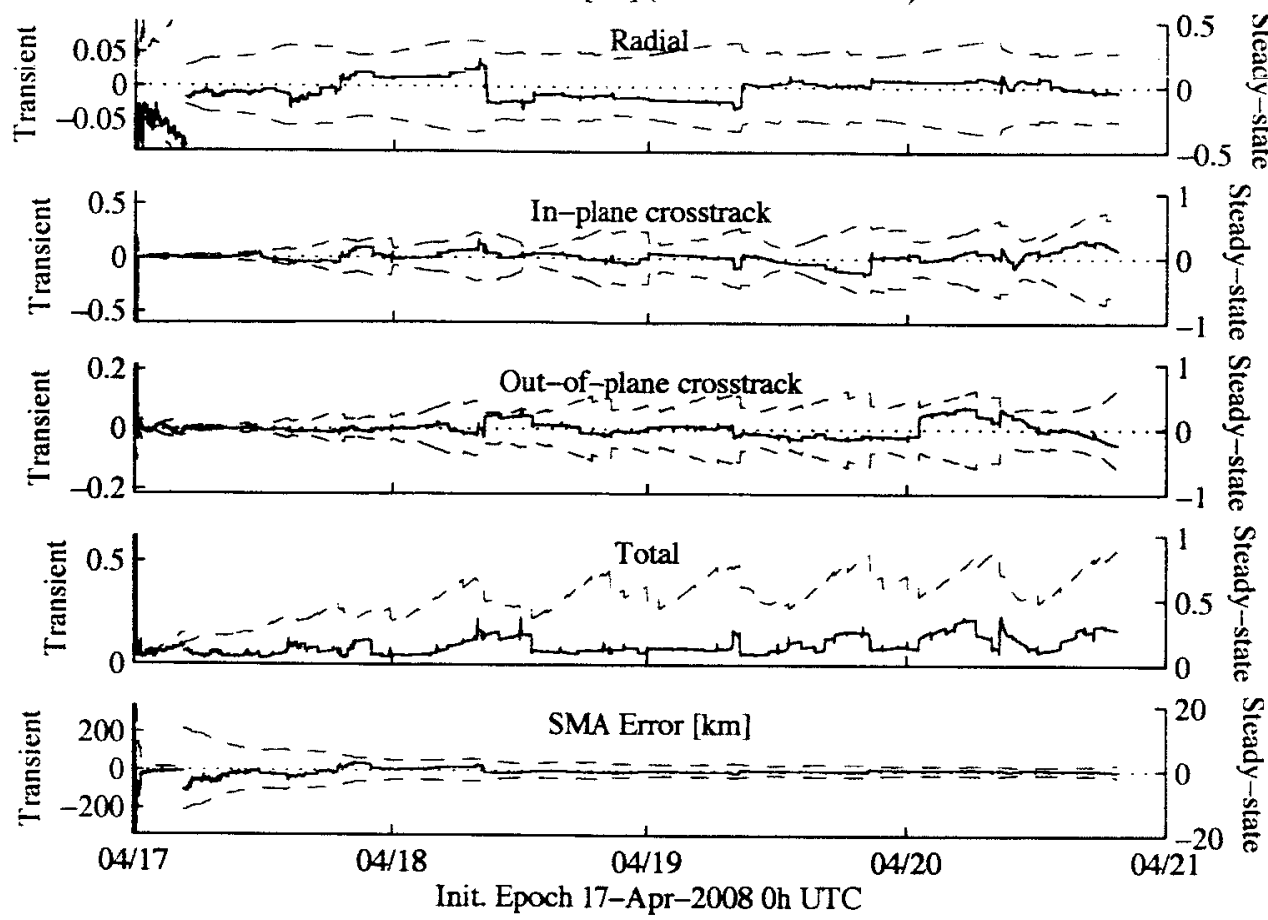

Figure 11. Transient (left axis) and "steady-state" (right axis) single-case position and semi-major axis (SMA) errors of the filter.
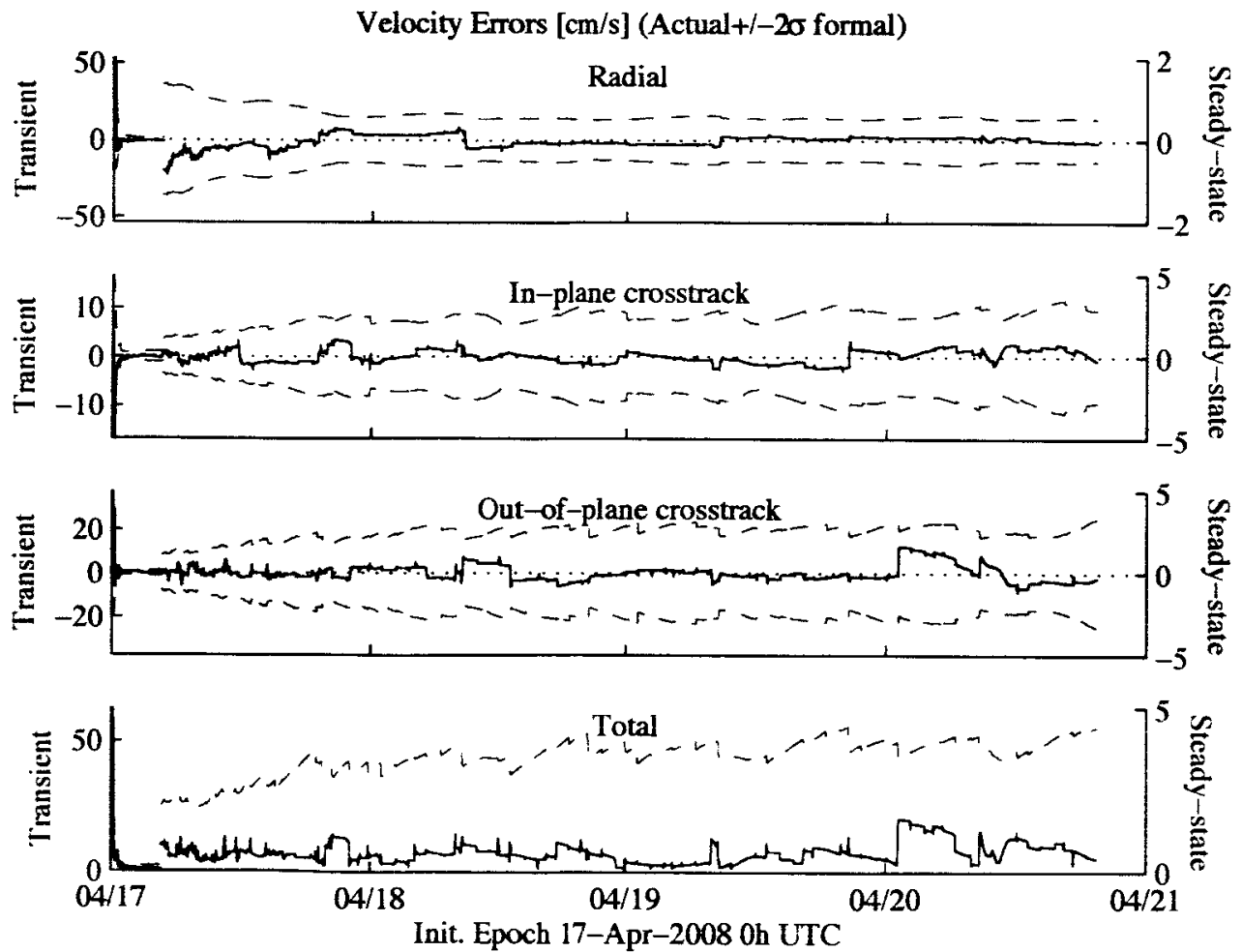

Figure 12. Transient (left axis) and "steady-state" (right axis) single-case velocity errors of the filter. 

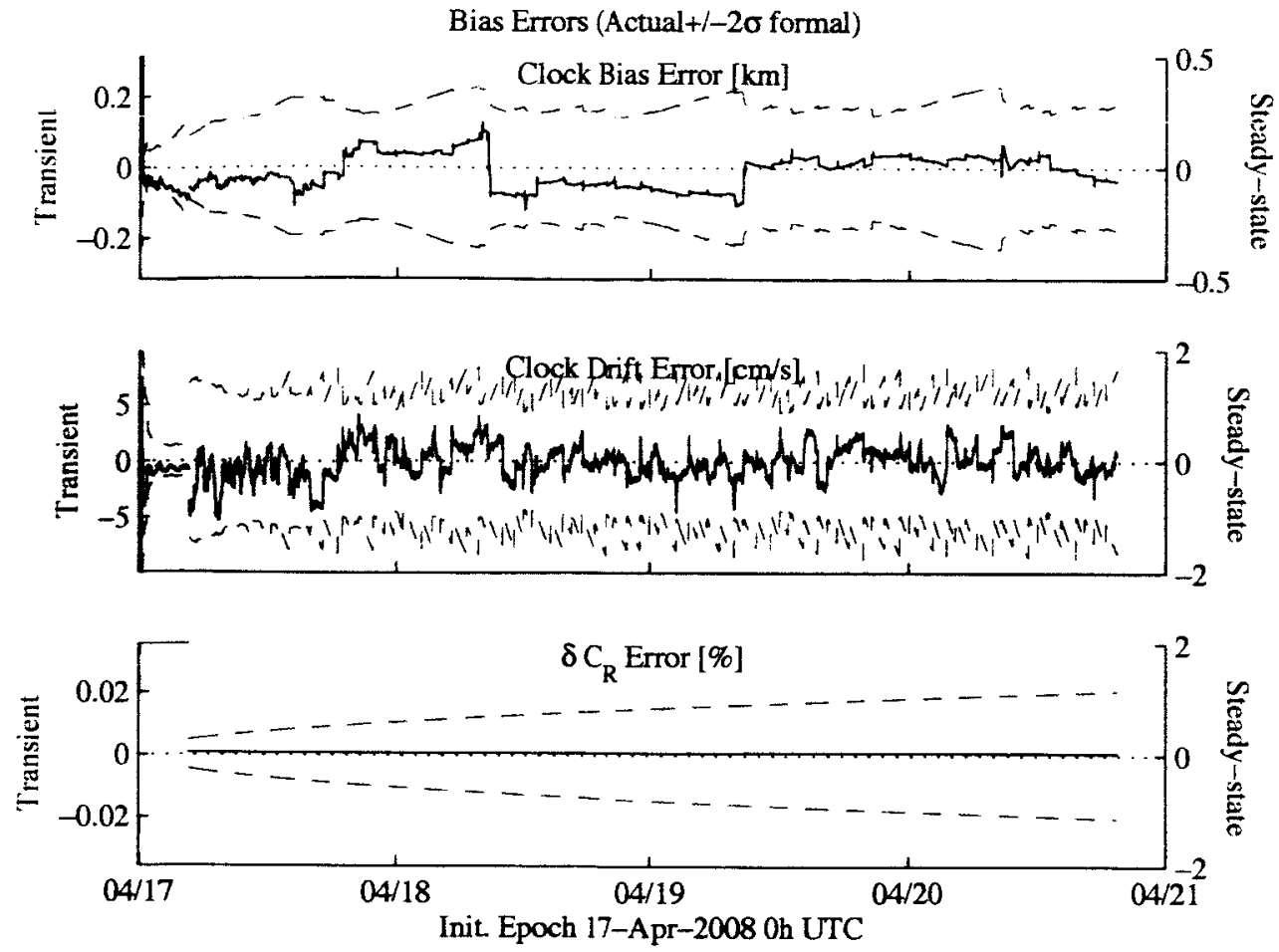

Figure 13. Transient (left axis) and "steady-state" (right axis) single-case bias errors of the filter.

\section{Summary and Conclusions}

In this work, we have examined the feasibility of an onboard navigation system for cis-lunar exploration, based on one-way Doppler from an Earth-Moon $L_{2}$ orbiter and GPS pseudorange measurements. Our study indicates that accuracies of better than $1 \mathrm{~km}$ and $5 \mathrm{~cm} / \mathrm{sec}$ may be feasible, which are quite promising in comparison with the trans-lunar post-maneuver results on the order of kilometers and dozens of centimeters per second achieved during Lunar Prospector using two-way Doppler from Deep Space Network 34- and 26meter tracking sites. Since these are single-case results, the value of the actual errors in predicting real-world performance is somewhat reduced. Instead, one may view these results as a guideline for the range of accuracy that might be achievable. Although we believe that a case can be made for lunar orbiting constellations in support of long-term human and robotic lunar exploration, we believe that the EML2 Orbiter provides a great deal of capability with minimal investment as a starting point for a comprehensive lunar and planetary navigation and communications infrastructure beyond near Earth orbit.

\section{Acknowledgment}

Frank Vaughn provided us with the graphics for Figures 1 and 4, and assisted with the development of the EML2 orbit concept.

\section{References}

${ }^{1}$ R. W. Farquhar, "The Control and Use of Libration-Point Satellites," Tech. Rep. NASA TR R-346, NASA Goddard Space Flight Center, 1970.

${ }^{2}$ C. A. Renault and D. J. Scheeres, "Statistical Analysis of Control Maneuvers in Unstable Orbital Environments," Journal of Guidance, Control and Dynamics, Vol. 26, No. 5, September-October 2003, pp. 758-769.

${ }^{3}$ D. C. Folta and F. J. Vaughn, "A Survey of Earth-Moon Libration Orbits: Stationkeeping Strategies and Intra-Orbit Transfers," to appear in Astrodynamics 2004, Univelt, 2004.

${ }^{4}$ G. L. Condon and S. W. Wilson, "Lunar Orbit vs. Libration Point and Lunar Surface Rendezvous Methodologies for Human Lunar Missions," Guidance and Control 2004, Univelt, 2004. 
${ }^{5}$ D. C. Folta, "Servicing And Deployment Of National Resources In Sun-Earth Libration Point Orbits," Praceedings of the World Space Congress, 2002.

${ }^{6} \mathrm{~K}$. C. Howell, "Representation of invariant manifolds for applications in three-body systems," to appear in Astradynamics 2004, Univelt, San Diego, CA, 2004.

${ }^{7}$ G. H. Barton, S. W. Shepard, and T. J. Brand, "Proposed Autonomous Lunar Navigation System," Astrodynamics 1993, Univelt, San Diego, CA, 1993.

${ }^{8}$ J. E. Draim, "Three- and Four-Satellite Continuous-Coverage Constellations," Journal of Guidance, Control and Dynamics, Vol. 8, No. 6, Nov-Dec 1985, pp. 725-730.

${ }^{9}$ T. J. Lang and J. L. Meyer, "A New Six Satellite Constellation for Optimal Continuous Global Coverage," Space Flight Mechanics 1995, Univelt, San Diego, CA, 1995, pp. 1451-1458.

${ }^{10}$ L. Winternitz, M. Moreau, and S. Sirotzky, "Navigator GPS Receiver for Fast Acquisition and Weak Signal Tracking Applications," to appear in Proceedings of the ION GNSS Meeting, Institute of Navigation, 2004.

${ }^{11}$ M. Psiaki, L. Winternitz, and M. Moreau, "FFT-Based Acquisition of GPS L2 Civilian CM and CL Signals," to appear in Proceedings of the ION GNSS Meeting, Institute of Navigation, 2004.

12P. S. Maybeck, Stochastic Models, Estimation, and Control, Vol. 1, Academic Press, New York, 1979.

${ }^{13}$ R. Gersten, "Statistical Properties of Orbit Perturbations Induced by the Earth's Anomalous Gravity," Journal of Spacecraft, Vol. 4, 1967, pp. 1145.

${ }^{14}$ J. R. Wright, "Sequential Orbit Determination with Auto-Correlated Gravity Modeling Errors," Journal of Guidance and Control, Vol. 4, No. 3, May-June 1980, pp. 304-309.

${ }^{15}$ G. J. Bierman, Factorization Methods for Discrete Sequential Estimation, Academic Press, New York, 1977.

${ }^{16}$ B. Hoffmann-Wellenhof, H. Lichtenegger, and J. Collins, Global Positioning System: Theory and Practice, SpringerVerlag, New York, 3rd ed., 1994.

${ }^{17}$ R. H. Battin, An Introduction to the Mathematics and Methods of Astrodynamics, ALAA, New York, 1987.

${ }^{18}$ The Astronomical Almanac for the Year 1984, US Government Printing Office, Washington, DC, 1984.

${ }^{19}$ IGeS Files Repository-JGM-2, http://www.iges.polimi.it/db/model/db.jgm2.html.

${ }^{20}$ T. C. V. Flandern and K. F. Pulkkinen, "Low-Precision Formulae for Planetary Positions," The Astrophysical Journal Supplement Series, , No. 41, November 1979, pp. 391-411.

${ }^{21} \mathrm{~S}$. Moshier, Astronomy and numerical software source codes, http://www.moshier.net/.

${ }^{22}$ I. Harris and W. Priester, "Theoretical Models for the Solar Cycle Variation of the Upper Atmosphere," Tech. Rep. TN-D-144, NASA Goddard Space Flight Center, 1962.

${ }^{23}$ D. A. Vallado, "Disturbing Forces," Fundamentals of Astrodynamics and Applications, chap. 8, Microcosm Press, El Segundo, CA, 2nd ed., 2001, pp. 508-548.

${ }^{24}$ W. M. Lear, "Range Bias Models for GPS Navigation Filters," Tech. Rep. JSC-25857, NASA Johnson Space Center, Houston, TX, 1993.

${ }^{25}$ M. Beckman and M. Concha, "Lunar Prospector Orbit Determination Results," Astrodynamics 1998, Univelt, 1998. 